Article

\title{
Body-Borne Computers as Extensions of Self
}

\author{
Sang-won Leigh *, Harpreet Sareen, Hsin-Liu (Cindy) Kao, Xin Liu and Pattie Maes \\ MIT Media Lab, Cambridge, MA 02139, USA; sareen@media.mit.edu (H.S.); cindykao@media.mit.edu (H.-L.K.); \\ xxxxxxin@media.mit.edu (X.L.); pattie@media.mit.edu (P.M.) \\ * Correspondence: sangwon@media.mit.edu; Tel.: +1-617-642-7996 \\ Academic Editor: Subhas Chandra Mukhopadhyay \\ Received: 31 December 2016; Accepted: 24 February 2017; Published: 9 March 2017
}

\begin{abstract}
The opportunities for wearable technologies go well beyond always-available information displays or health sensing devices. The concept of the cyborg introduced by Clynes and Kline, along with works in various fields of research and the arts, offers a vision of what technology integrated with the body can offer. This paper identifies different categories of research aimed at augmenting humans. The paper specifically focuses on three areas of augmentation of the human body and its sensorimotor capabilities: physical morphology, skin display, and somatosensory extension. We discuss how such digital extensions relate to the malleable nature of our self-image. We argue that body-borne devices are no longer simply functional apparatus, but offer a direct interplay with the mind. Finally, we also showcase some of our own projects in this area and shed light on future challenges.
\end{abstract}

Keywords: wearable devices; symbiotic interfaces; on-skin interfaces; augmented human

\section{Introduction}

The ubiquity of information technology no longer separates us from technology. Clark argues that human beings are already cyborgs [1], as they are innately driven to use the environment and external tools as extensions of themselves [2]. A classic example mentioned by Clark is timepieces. When you ask if a person knows the time, that person would answer "yes" even without knowing the time and then check his/her watch. As such, we offload our cognitive processes to artifacts around us [3], and for centuries we have extended our capabilities through similar means of incorporating technologies.

Exoskeletons [4,5] or prostheses [6-8] are well known examples of the integration of humans and machines for improving personal abilities. Sensory substitution $[9,10]$ is a field of research aiming to supplement a loss of sensory modality with another, or to enable the perception of signals that we normally are not capable of sensing. While a variety of related subjects have been presented in the field of human-computer interaction ( $\mathrm{HCI})$, these kinds of work are distinct from traditional user interface (UI) [11] research. In contrast to UIs that temporarily provide abilities to a user, augmented human (AH) technologies are designed to be always available and operating for an extended period of time. They also often establish direct interfacing with the body and are, thereby, not limited to control by our fingertips.

This poses the questions of how a technology that is tightly coupled with the human cognitive or physiological system will be perceived and where the boundary lies between the technology and the human. The field of embodied cognition $[12,13]$ hints at the transformative potential of the human body image for incorporating extended, or alternative, capabilities. On a similar note, Lanier, in his pioneering virtual reality (VR) works, proposes the idea of homuncular flexibility (HF) $[14,15]$. He argues that human brain plasticity allows for the remapping of the body's motor control to a different, non-anthropomorphic body (also see [16]). In his collaborators' and his recent VR user study [17], it is reported that, within a few minutes, participants were able to learn how to control 
avatars with non-anthropomorphic (having a third arm) or juxtaposed (e.g., the legs controlling the hands of an avatar) configurations.

In this paper, we offer a perspective on future $\mathrm{AH}$ designs that facilitates a stronger interplay between the body and mind, through directly changing the body's morphology or visual/sensing capabilities. We discuss how the use of a tool not only helps achieve a task but also changes the way we perceive ourselves and the body's functionalities. It is well known that the possession of an opposable thumb, as well as the skills of utilizing tools, resulted in a significant difference between humans and other species on Earth. The acquisition of sophisticated manual skills accompanied the growth in brain size of Homo sapiens, signifying the effect of corporeal capabilities on how the brain develops $[18,19]$. This effect is not limited to genetic modifications; modern neuroscience research suggests that our internal body model updates, given temporary extensions to sensorimotor capabilities [20-23].

In light of this discussion, we will review three areas of augmentation of the human body: physical morphology, skin display, and somatosensory extension, along with a framework that puts works from different research domains into perspective. We add some of our ongoing research progress in the discussion, and show how the HCI approach can extend the research with more computing, interactivity, and application perspectives. Finally, we identify future challenges and what opportunities there are within the HCI domain.

\section{The Human Body in HCI}

Although the human body plays an important role in HCI, explicit discussions of the role of the body did not happen until the late 1990s [24]. Gaver's take on affordances [25] is also largely focused on media spaces, whereas the original concept by Gibson [26] describes the relation between a living organism and its environment. Such a tendency to look away from the body tracks back to Heidegger's Being and Time [27], which only has six lines about the body.

On the other hand, insights into the role of the body in interactions can be found from Merleau-Ponty's works [28] on the embodied basis of human perception. He rejects the idea of perception as passive reception of a stimuli, and sees that there is no perception without action; active perception being one of the core ideas in embodied cognition research [29]. In other words, the condition of the body (such as past experiences or trainings) determines the way a person perceives. The effect of the body on perception is denoted as the "phenomenal field" by Merleau-Ponty [28].

Neuroscience studies reveal evidence that tool-use induces changes in how the brain processes the body image [21,22]. When a macaque monkey uses a rake for collecting food, its visual receptive field is enlarged to include the rake [21]. A continuous tactile stimulation can lead a person to have the sensation of having a third arm, even feeling touches on both a rubber hand and a biological hand [30]. Aymerich-Franch [23] proposed an overarching hypothesis relating to the neural phenomena of self-attribution-“our brain attributes a perceived entity as our limb, if the physical properties of the entity are sufficient to afford certain actions the brain has associated with that limb."

\section{Symbiotic Human-Machine Interfaces}

The relationship between brain plasticity and tool-use hints at a new perspective on how computational systems can be designed to form a stronger bond with users. Tools, wearables, and prosthetic devices do not just offer functional capabilities, but can be designed to become an integral part of the human system. We envision such technologies to offer extended functions of existing parts of the body, and establish low-level communication with our sensorimotor or somatosensory systems. Therefore, a body-integrated (-worn or -implanted) technology has the potential to be perceived as an intimate and natural extension of ourselves.

A well-known example is a study by Bach-y-Rita [10], where a vibro-tactile feedback is used on the back of visually impaired subjects to give them an alternate way of perceiving images. A noteworthy part in their report is: "...subjects spontaneously report the external localization of stimuli, in that sensory information seems to come from in front of the camera, rather than from the vibro-tactors on 
their back." Their system does not only provide an alternative channel for visual perception, but the entire action-perception loop is reconfigured and encoded in the subjects' cognitive architecture.

The concept we propose is a continuation of Licklider's vision [31], where humans and machines synergistically collaborate on accomplishing tasks by offloading repetitive and mechanical procedures to the machines. With the advances in computing, robotics, and bio-electronic technologies, it is possible to realize a much closer and low-level cybernetic loop between humans and computers that goes well beyond a simple role division. In later sections of this paper, we will discuss a larger body of related works that aim to realize such forms of human-computer integration.

\subsection{In Relation to Traditional HCI}

The field of HCI has so far explored two major paradigms for how people relate to machines. The dominant approach is that of the machine as tool exemplified in the widespread use of graphical user interfaces (GUI) [11], as well as the emerging category of tangible user interfaces (TUI) [32]. The interaction consists of the user employing software or hardware tools to get things done. A second approach, which has recently gained a lot of attention, is that of the machine as an intelligent agent. Interactions in this case typically involves commands or conversations in natural language, and the Holy Grail is for the machine to become an autonomous worker as intelligent as a human.

The third approach (Figure 1) we discuss in this paper consists of an intimate integration of human and machine. On the one hand, it attempts to mitigate the two existing paradigms by offering semi-intelligent systems interfaced with the human body so that they become always-available tools for sensing, controlling, and performing actions. On the other hand, the semi-intelligent system and the user become integrated, with the system forming a natural extension for an augmented human. The form of such a realization will often be, but not limited to, wearable, implanted, or skin-attached systems.
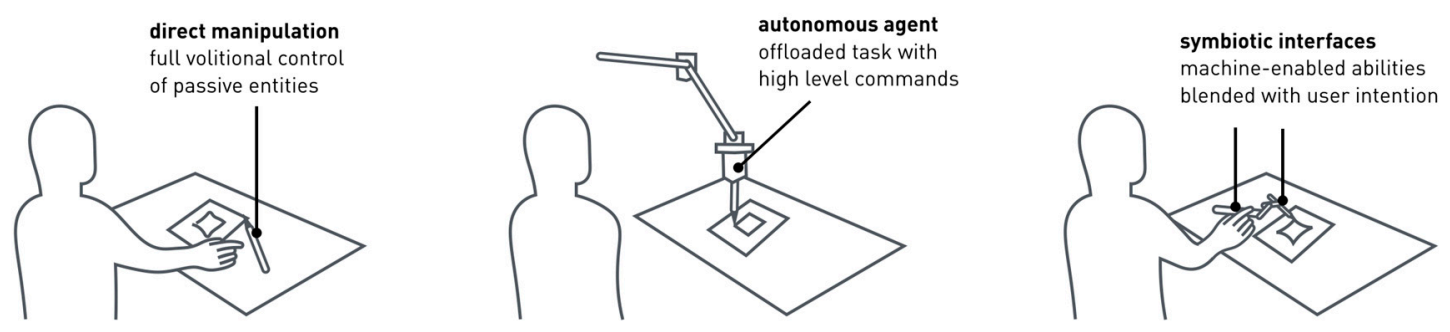

Figure 1. Symbiotic interfaces combine aspects of the two prior interaction paradigms (direct manipulation and autonomous agents).

\subsection{Implications for Designing User Interfaces}

Neuroscience studies provide evidence of how tool-use or repeated behaviors can lead to different paths of neural development. For example, a skillful use of the hand changes the cortical activity allotted to the tactile receptors on fingertips [33-35]. It is reported that string instrument players have larger somatosensory cortical activity in response to touch on the little fingertip than non-players [34,35]. Recently, scientists showed that the cortical potentials from the thumb and index fingertips were directly proportional to the intensity of smartphone use [33].

These findings point at the role of UI design in affecting development of new neural circuitries and mappings. The change in users is not limited to tactile receptors; studies of neural prostheses [8] showed developments of new muscle synergies through continuous training on myoelectric controls. Cognitive science studies further demonstrate changes in perception and behavior of users as a result of giving users VR avatars with different traits, such as gender [36], body shape [37], or character [38]. Despite the evidence, current interaction or interface designs rely heavily on the innate skills users possess, or their natural body image, meaning that they regard the user as a time-invariant parameter. However, interfaces can be designed to co-develop with the user's physical or cognitive abilities. 


\section{Symbiotic Interfaces that Extend the Self}

In this section we divide possible augmentation technologies into subcategories: (1) physical morphology—sensorimotor augmentations that act as end effectors or sensory probes that offer functional extensions of the body; (2) skin display-augmentations that extend the aesthetic, informational, or sensing capabilities of our skin; and (3) somatosensory extensions-means to create computational alterations of perception for pseudo-nervous mappings (Table 1). We exclude discussions on general sensory substitution in this section, focusing on ones that modulate or extend the sense of self.

Table 1. The categories of symbiotic interfaces discussed in the paper.

\begin{tabular}{|c|c|c|c|}
\hline Physical Morphology & Skin Display & Somatosensory Extension & Reconfiguring Biological Circuit \\
\hline $\begin{array}{l}\text { Through change in structural } \\
\text { or material properties }\end{array}$ & $\begin{array}{l}\text { For self-expression or } \\
\text { biosignal visualization }\end{array}$ & $\begin{array}{l}\text { Through interoceptive or } \\
\text { exteroceptive interventions }\end{array}$ & \multirow[b]{2}{*}{$\begin{array}{l}\text { Biological intervention } \\
\text { into the human system. } \\
\text { This category includes implants } \\
\text { and surgical modification of the } \\
\text { human physiology. }\end{array}$} \\
\hline $\begin{array}{l}\text { Neuromuscular Control: } \\
\text { Establishing a new } \\
\text { communication between the body } \\
\text { and the brain }\end{array}$ & $\begin{array}{l}\text { Bio-sensing: } \\
\text { For collecting biosignals } \\
\text { from the body }\end{array}$ & $\begin{array}{l}\text { Addition of New Sensations: } \\
\text { Through creating new } \\
\text { forms/combinations of } \\
\text { sensory feedback }\end{array}$ & \\
\hline
\end{tabular}

The division into the three categories is defined based on the traditional role of the body-its mechanical capability, use for expression, and sensing capability. The physical shape and mechanical structure (e.g., muscular) defines the physical capabilities afforded by a body part or the body as a whole. The surface of the body affords interactions with the environment or in social contexts. It has been used as a display (for self-expression in the form of makeups and fashion), sensory organ (texture, temperature, humidity, and so on), and a place from which to acquire biosignals (heartbeat, body temperature, and so on). Finally, events or feelings (somatosensory) from the body critically determine emotions or the perception of self. In order to illustrate the three spaces for augmentation, we introduce works from various domains including some of the authors' own works.

We also include a section on reconfigured bio-electronic circuits in living organisms. This separate section intends to look into further possibilities through more invasive modifications of the body. Such techniques are also often used as complementary elements to the other three categories, e.g., for sensing and transmitting biosignals through the body.

\subsection{Physical Morphology}

This section discusses augmentation technologies that provide functional morphology change. The augmentation may offer increased sensorimotor capabilities to a user [39-43] or exploit existing body structure $[44,45]$ for added functionalities. "Morphology" in this section can be (1) the physical shape of the body or the material/functional property of the extremities (external morphology); or (2) how the body and the brain communicate for motor control (internal morphology).

\subsubsection{The Shape of the Body—External Morphology}

Unlike the commonly known type of exoskeletons—parallel-limb exoskeletons [46,47]—series-limb exoskeletons [48-50] offer interesting extensions to their wearers [51]. They are designed to be connected with a user in series, extending or transforming the extremities of the user to provide new properties. PowerSkip [49] is a wearable device that provides leg extensions that help a user jump extremely high, and SpringWalker [50] is a contraption that allows faster and more economic maneuvering with longer legs. Gloves used by NASA [52] change stiffness so that their users can effortlessly hold heavy objects, and gecko-inspired climbing gloves [53] give its wearer a temporary ability to climb on vertical walls. 
Supernumerary robotics (SR) [39-41] is a recently established field of research that studies additional robotic fingers or limbs that support physical tasks by reducing load or aiding in object manipulation. SR fingers [40,41] are designed to work in synergy with a user's fingers, offering improved hand grasp capabilities. Shoulder-mounted SR arms $[39,54]$ are designed to support their wearer in accomplishing assembly jobs that would normally require two men with extra robotic arms instead. Similarly, a pair of robotic legs [55] allows the user to maneuver on extreme terrain and balance better. Georgia Tech demonstrated a three-armed drumming system [56] where the high-level control of the robotic arm is performed by brain signals and software that fine-tunes the rhythm to fit into the music being played.

Seminal art works by Stelarc, such as The Third Arm [57], are muscle-controlled robotic contraptions worn on the body. Through the muscles around his abdomen, the artist was able to control the third arm, along with two biological ones, and write characters with all three arms at the same time. Horn, in her art pieces Pencil Mask (1972) and Two Hands Scratching Both Walls (1974-1975), showcases structures worn on the body that heighten the wearer's senses.

\subsubsection{Control of the Muscles through Computers-Internal Morphology}

Existing limbs may also be used and controlled through computational inputs-a computational device acquires the capability to drive muscles, e.g., independent from a user's intention. Furthermore, the computational control of muscles can allow new synergies between individual muscles, which is not possible with our internal neuromuscular structure. Tokyo University showcased the use of functional electrical stimulation (FES) as a means of controlling the human body [44], allowing a person to control another user's body as a surrogate for teleoperation. Inferno [58] is an art performance in which participants are asked to wear robotic exoskeletons and are controlled/forced to dance in synchronization. In other words, a user's body is temporarily controlled by another human or a machine operator.

\subsubsection{Sensorimotor Retargeting}

Sensory channels combined with motor actions allow active perception-like turning the head to see from different perspectives, therefore, better spatial cognition. An addition to new sensing capabilities to a body part may open up opportunities for new sensorimotor mappings between action and sensing. In contrast to the external morphology section, sensorimotor retargeting focusses on the data transmitted or received in accordance to motor actions.

A study by Bach-y-Rita [10], mentioned earlier in this paper, reports that people perceive visual feeds from a camera through tactile feedback on their back as coming from in front of the camera, not from their back. Subdermal implantation of a magnet has also been studied to explore sensory alterations of the fingertips [59]. FingerReader [42] is a camera worn on a finger that reads texts shown to the camera to its wearer. Effectively, the finger turns into an information probe where a user can move and point in different directions to read spatially-relevant information. Such a connection between motor behavior and sensing has been explained by Haans and IJsselsteijn: "When the mediating technology allows for these sensorimotor contingencies to be registered, or enacted, one will experience the digital content as having a specific physicality in time and space" [60].

The body can also be modified to output data. Warwick [61] implanted a microchip in his wrist that makes contact with the nerve bundles, where the nervous signals are read and transmitted to computers. Ishin-Den-Shin [43] is a wrist-worn device that conducts sound through bones, enabling one to send audio messages to another person by gently tapping on the ear.

\subsubsection{More Programmability in the Body}

Research in shape-changing robotics hints at how we can further extend the adaptability of our body shape and, therefore, our physical capabilities. We have developed a set of wrist-worn programmable robotic joints that offers additional action capabilities to users [62] (Figure 2). The main 
motivation is to create a modular system of which the functionality can be reconfigured in a more "programmatic" manner, and offer a platform through which a multitude of applications can be realized. The application includes modes where the joints act as extra actuators (or fingers), passive support structure (such as for holding a notepad for one-handed note-taking), or haptic user interfaces for VR games. Different modes of operation require different controller schemes, however, the input signal used for the control remains unchanged. In other words, software modifications alone can enable the user to have interchangeable (robotic) functions controlled by the same set of muscle movements.
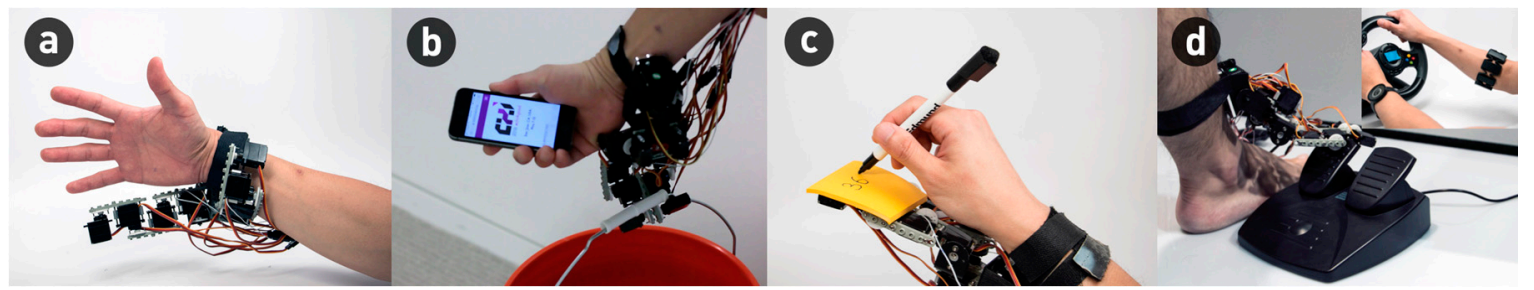

Figure 2. Body-worn robotic device that transforms into multiple forms.

Modularity in a morphology-extending prosthetic device can further improve the adaptability of such technologies. We are developing a modular assembly kit consisting of basic sensor or motor components that somewhat resemble those of human joints (Figure 3). The modules, with a universal connector design, can easily be arranged in different orders-like we arrange different programming blocks in software development. To date, we implemented servomotor modules with two different axes of rotations, as well as sensor modules so an engineer can add new sensing capabilities of choice to the module chain. Customization of end-effectors is possible through 3D printing pluggable fingertip modules, allowing the robotic device to have effectors designed for specific tasks, such as for plucking guitar strings (Figure 4).

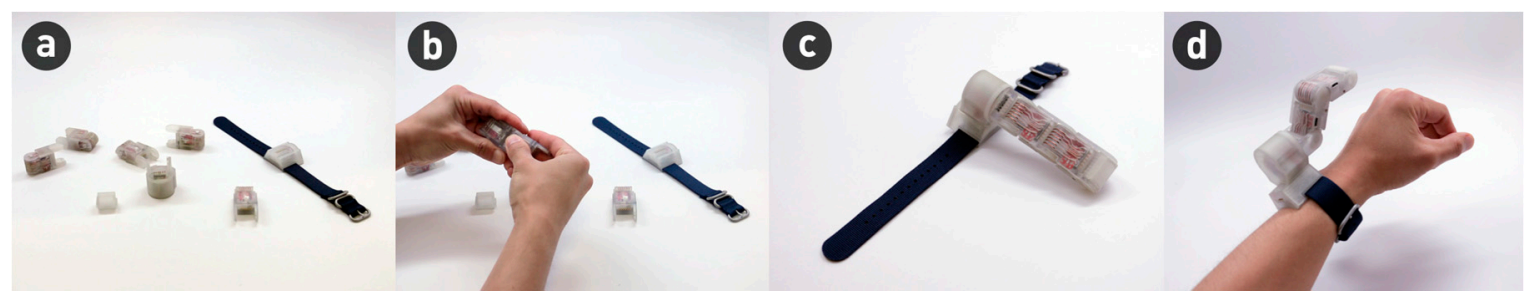

Figure 3. Modular robotic wearable system: (a) modules for robot assembly; (b) the modules are connected by a single clasp action; (c) an assembled robot connected to the wrist attachment module; (d) the robot in action, worn on a user's wrist.

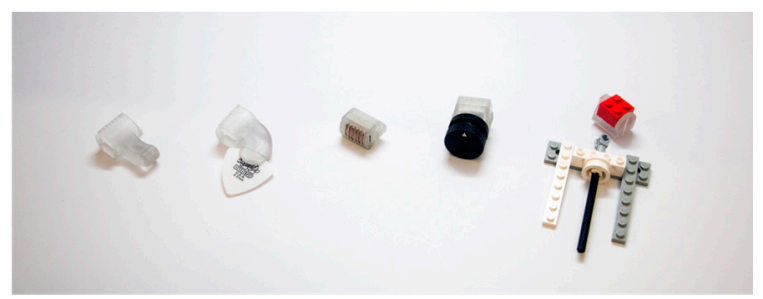

Figure 4. Different end effector module designs-(from left to right) trigger, pick holder, sensor tip, knob tip, and LEGO connector.

\subsection{Skin Display}

Emerging work at the intersection of fashion and technology has explored skin and interactive garments as displays of information or the wearer's emotional state [63-67]. Several interactive wearable 
garments visualize the wearer's discomfort towards violation of personal space. For instance, Caress of the Gaze [63] morphs in response to unwanted visual attention. Amisha Gadani [68], Meejin Yoon [69], and Anouk Wipprecht [70] created defensive dresses that exhibit animal-like behaviors in response to fear upon the encroachment of personal space. These works are all examples of social prostheses that amplify the automatic emotional response a wearer may exhibit.

Other works, such as Ying Gao's Incertitudes garments [71] are integrated with motorized pins that shift according to the spectators' voice to explore ambiguity in conversation. Liu and Lengeling [72] presented a speculative design of artificial goosebumps that reflect a user's stress levels. Their idea is inspired by the Iowa Gambling Test [73], a study that shows that a subliminal "stress" signal is produced by the body even when the person is not consciously aware of the stressful situation.

The examples above illustrate the opportunities of augmenting skin in three application areas-display, bio-sensing, and reaction to external stimuli. The skin has not only been used for curating identity, but is also innately responsive to the environment (e.g., temperature, humidity) and channels emotional responses through visual or physical changes of the skin.

However, most of the HCI research to date has focused on on-skin interactions for touchscreen input styles, or techniques for fabricating microcircuits in a small form factor to be attached to the skin [74-78]. We briefly discuss prior research and present three research studies that describe how further incorporation of a biochemical approach can create a more skin-friendly skin augmentation products, with customized bio-sensing or environmental sensing capabilities.

\subsubsection{Techniques for Skin Augmentation}

HCI researchers have used bio-acoustic [74], capacitive [75,76], and magnetic [77] sensing to appropriate the human skin as input surface. In order to use on-skin surfaces for visual output, researchers have explored mounting small projectors [74] or displays [78] onto the body. The Vivalnk tattoo, a commercially available tattoo sticker, has near-field communication (NFC) capability [79]. For a more in-depth review of wearable designs for skin interfaces, please refer to [65].

The most critical challenge in designing on-skin interfaces is the minimization of electronics and form factor. In order to address this, several fabrication processes have been proposed. In material science, epidermal electronics integrated with soft, stretchable forms that have skin-like properties have been explored [80]. However, incorporating sensing capabilities with such form factors poses challenges in manufacturing and cost $[81,82]$. More widely accessible fabrication processes have been proposed as well. iSkin is a silicone-based skin overlay for touch sensing [76], however, it requires the use of material-science grade materials (PDMS with carbon) and its thickness of $700 \mu \mathrm{m}$ is much thicker than that of epidermal electronics $(0.8 \mu \mathrm{m})$. Skintillates prints conductive silver ink on temporary tattoo paper to create $36 \mu \mathrm{m}$ thick on-skin electrical traces [66].

\subsubsection{Chemically-Produced Interactive Skin Interfaces}

SkinSense [65] is a chemically-produced tattoo-like interface that has a layer of stretchable electrodes (Figure 5). Components, such as sensors or LEDs, can be connected, enabling designs of interactive tattoos. An example is composed of RGB LEDs and IR receivers, therefore, a facial tattoo can be controlled by a remote controller for interactive play and aesthetics purposes. DuoSkin [64] is a fabrication process that generates ultra-thin functional devices which can be attached directly onto the skin. It leverages gold metal leaf, a material that is cheap, skin-friendly, and robust for everyday wear. By overlaying multiple layers of gold metal leaf, DuoSkin devices can function as a capacitive touchpad or as an antenna for wireless communication (Figure 6). Importantly, DuoSkin incorporates aesthetic customization similar to body decorations, giving form to exposed interfaces that so far have mostly been concealed. 


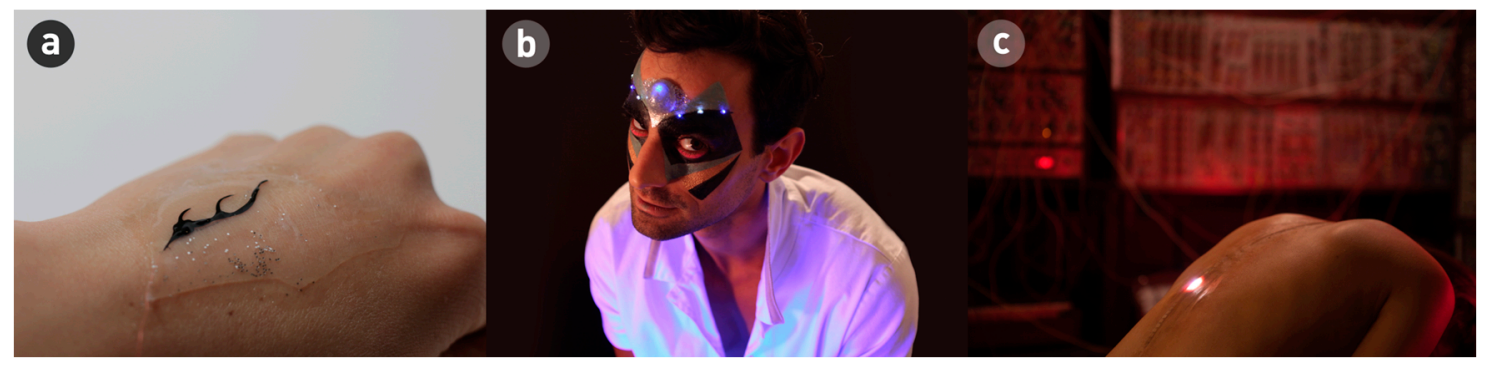

Figure 5. (a) SkinSense offers stretchable electrode in a tattoo-like form; (b,c) different aesthetic implementations of SkinSense.
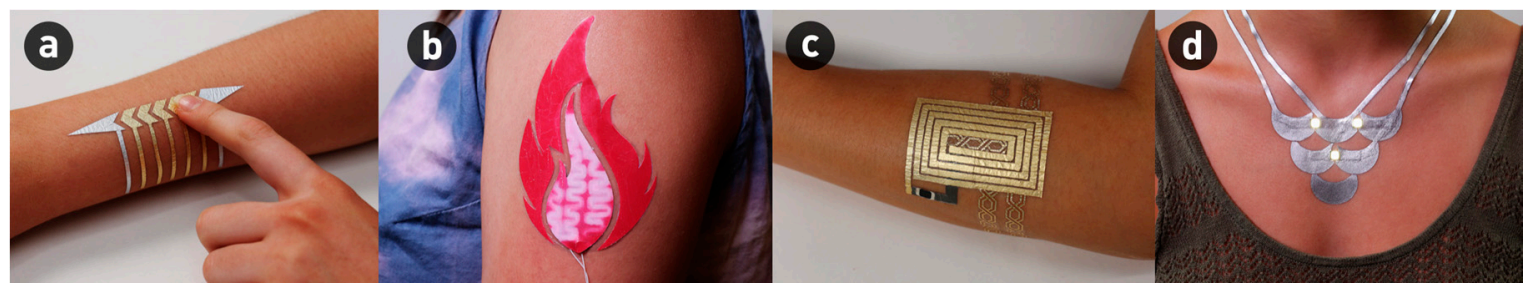

Figure 6. DuoSkin: (a) touchpad application; (b) thermochromic display; (c) NFC antenna; and (d) interactive garment.

A chemically-produced interactive tattoo can offer minimal and specific-purpose computing powers to the skin (Figure 7). Due to its spatial affinity to the skin, such tattoos can acquire biosignals from the body or respond to environmental conditions to inform a user of potential risk. In the project Dermal Abyss, biochemical sensors are used to acquire and display information about the physiological state of the user. The biosensors developed are capable of indexing the concentration of sodium, glucose, and $\mathrm{pH}$ in the interstitial fluid of the skin, and output the collected data through colors on a tattoo. Currently, the experiments are conducted ex vivo using a pig skin due to clinical experimentation challenges.

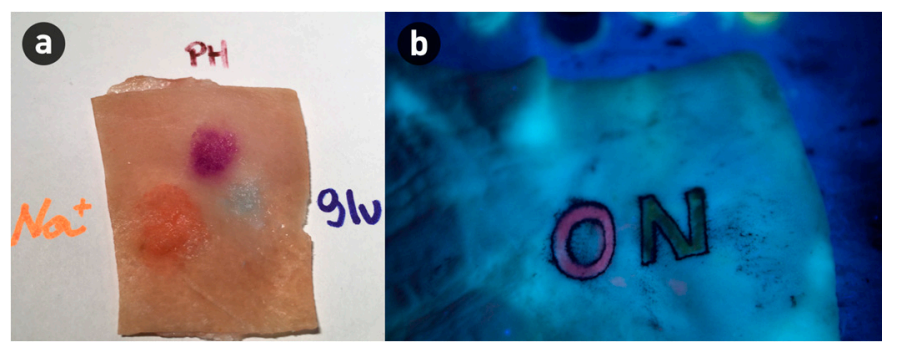

Figure 7. (a) Biosensors tattooed onto pig skin and their interaction with chemical solutions; and (b) fluorescent biosensors made by a professional tattoo artist on pig skin.

EarthTones [83] is a cosmetics-inspired wearable chemical sensing powder to detect and display environmental conditions (Figure 8). It presents an alternative to current mainstream wearable displays (e.g., smart watches, LED textiles), which are digital and battery-laden. Instead, EarthTones present a direction towards soft, analog wearable displays composed of chemical-sensing powders for increased wearability, which are also built upon habitual makeup practices. The powders detect elevated levels of carbon monoxide (CO), ultraviolet (UV) rays, and ozone $\left(\mathrm{O}_{3}\right)$ and trigger corresponding color change to increase environmental awareness. 


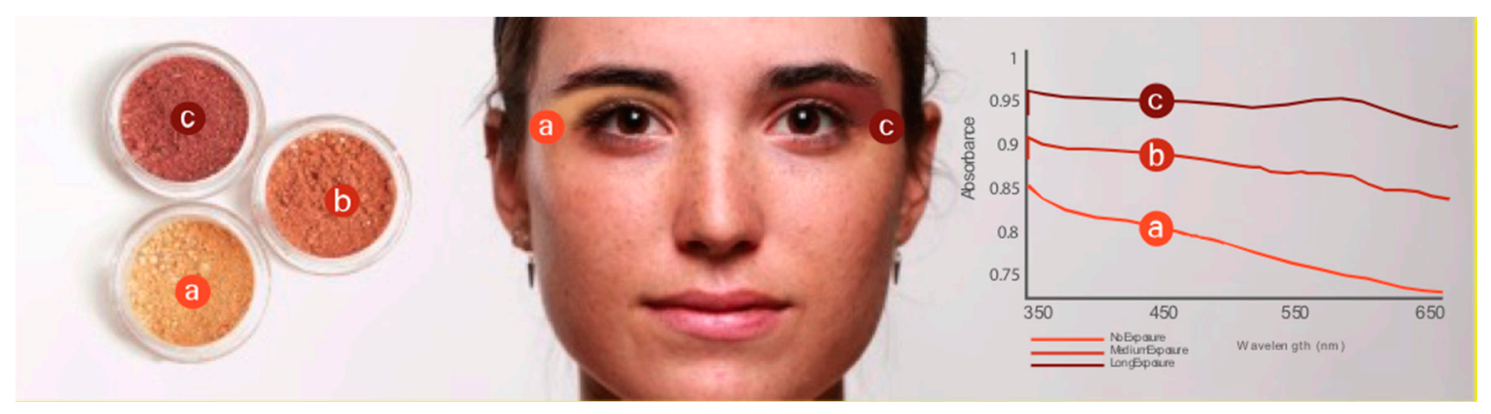

Figure 8. EarthTones is a wearable chemical display in the form of cosmetic powders. It senses environmental pollution, and generates color change to display elevated levels. We created three powder instantiations which detect carbon monoxide $(\mathrm{CO})$, ultraviolet radiation (UV), and ozone $\left(\mathrm{O}_{3}\right)$. In the example of a UV-sensing powder, a color change from yellow (a) to dark red (c) occurs when exposed to UV.

\subsection{Somatosensory Extension}

In this section we discuss the third category of sensorimotor human augmentation namely somatosensory extensions. We distinguish our scope from the large body of sensory augmentation research that aims to substitute or add new sensory modalities. Instead, we focus on ones that re-configure how we interpret biosignals or the body image to include external entities as a part of oneself. We discuss interoceptive and exteroceptive means of "deceiving" the perception of self or of internal biosignals, and introduce a work in development that aims to go beyond relying on existing interoceptive cues.

\subsubsection{Interoceptive Modulation}

The phenomenon of gut instinct reveals a sensorial path from the inside out, where feelings are directed toward the external world. A work from Critchley and colleagues [84] suggests that activity in the right anterior insular/opercular cortex predicted subjects' interoceptive accuracy, which correlates to awareness of emotions. For example, a person may perceive themselves to be anxious if they notice elevated heart rate. Interoceptive sensitivity and accessibility are further proven to correlate with self-awareness, wellness, and social behaviors [85].

There are very few interactive applications that utilize interoception, however, in the field of medical and psychological therapies [86-88] interoceptive exposures have been widely used for mitigating hypersensitivity to internal alerts. Interoceptive exposure (IE) is a procedure in which a patient goes through a set of exercises to produce a sensation that is close to what causes panic disorder [86,87], irritable bowel syndrome [88], or another problematic condition. In that way, the patient becomes desensitized to the interoceptive cues, thereby effectively removing the trigger for certain disorders.

Modulation of interoceptive signals has also been studied in VR environments. A study suggests visual stimuli that are close to one's heartbeat signal can alter the perceived self-physiology [89]. Although the study's aim is to affect interoception through non-interoceptive stimuli, it suggests that, in immersive environments, such techniques can be used to attribute an emotional presence beyond visual manifestation in a virtual environment.

\subsubsection{Exteroceptive Modulation}

There are well-known examples that use multisensory stimulation to alter body representation, such as the rubber hand illusion [30], and mirror-touch synesthesia [90]. An experiment by Tajadura-Jiménez [91] shows that the representation of key properties of one's body, like its length, is affected by the sound of one's actions. Recent studies have shown that synchronous interpersonal multisensory stimulation (IMS) produces quantifiable changes of mental representation of one's self 
and dilutes the differentiation with others [92]. BeAnotherLab [93] created a project called The Machine To Be Another that uses virtual reality to encourage empathy by swapping the user's body with the body of another gender.

\subsubsection{Novel Somatosensory Experience}

A somatosensory intervention opens up opportunities for creating new sensations by triggering sensory receptors programmatically. Such possibility can particularly reinforce immersion with virtual avatars, where an embodiment into avatars different from the self can potentially accompany corresponding somatosensory feedback.

TreeSense [94] is a tactile VR experience of being a tree (Figure 9). Through a headset, one can see their body in a form of a tree with two main branches moving along with their arms. In addition to that, a pair of EMS devices on each arm triggers "novel" tactile sensations that are not innately possible. The triggered sensations are the result of tactile induction and actuation of multiple muscles at varying intensities, in a way that will not happen normally. Therefore, a feeling of something crawling on the body or growing from inside the body can be simulated.

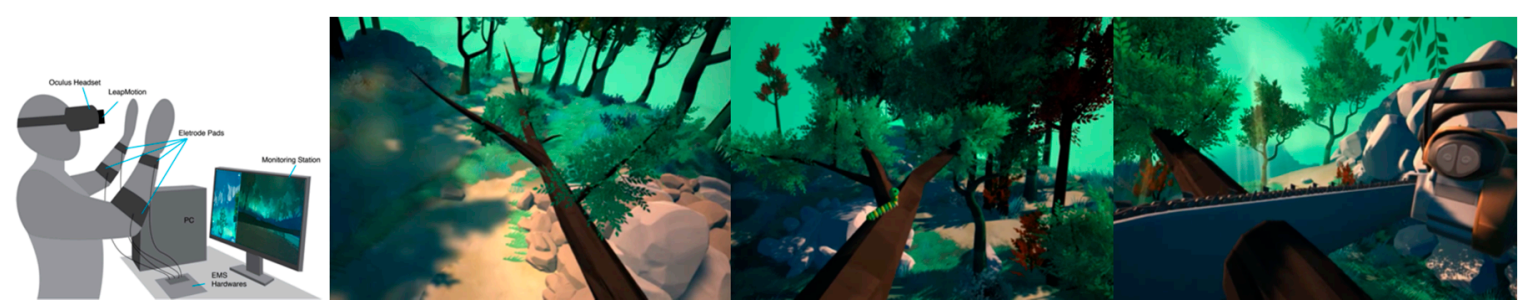

Figure 9. Scenarios of interacting with a non-anthropomorphic body (a tree). (a) EMS setup; (b) branch growing; (c) caterpillar crawling; and (d) getting chopped down.

The focus of this work is on somatosensory interventions through the electrical actuation of internal parts of the body. However, other sources, such as mechanical vibration, sound, or electronic implants, could expand how novel somatosensory experiences can be created. The use of programmatic induction of new somatosensory cues can be utilized for natural modalities for informing a user of their physiological state, or for virtual body ownership in VR with non-human configurations.

\subsection{Reconfiguring Biological Circuits}

In this category, we discuss research on computationally-augmented biological circuits. We focus on examples demonstrating practical applications, such as neural prostheses, in order to illustrate possibilities outside laboratories.

Advancements in electronics now afford a direct interface with biological systems. While pacemakers and defibrillators were initial instances of bio-hybrid systems, a new convergence of computation with biological substrates is on the horizon. Adamatzky and colleagues showed various functional biomorphic computing devices operated by slime mold [95]. In the same vein, mobile robots interfaced with brain cells of worms [96], rats [97,98], and monkeys [99] were proposed by various researchers.

But these explorations are not limited to lab experiments, there have been many clinical studies regarding the treatment of peripheral nerve injuries that cause problems, such as loss of function or neuropathic pain [100]. Neural prostheses [8,101,102], and cell or tissue transplants [100,103,104], have employed direct interfacing with the internal physiology for treatment. Osseointegration [101] is a technique that connects electrodes directly through a socket anchored through the bone. Thereby, nerves and muscle bundles can be accessed internally, reducing noise and instability of acquired biosignals. It is further reported that tactile perception could be chronically reproduced through direct electrical stimulation of the peripheral nerves, despite long-term amputation (>10 years). Targeted muscle reinnervation (TMR) [105] is a surgical procedure for retargeting remaining arm nerves to 
alternative muscle sites, such as remaining upper-arm or chest muscles. Therefore, loss of muscles due to an amputation can be overcome, and produce a sufficient number of signals for neuroprosthetic control. Researchers also explored producing axons in vitro, and implanting those bioartificial axon modules to bridge nerve damage $[100,103,104]$. The modules can include electrodes exposing electrical contacts to the subdermal areas, making nervous signal readings and inputs accessible [103].

\section{Discussion}

In this paper, we introduced works from various domains in order to shed light on the rise of human augmentation technologies. The paper highlights the interdisciplinary nature of the research and how the HCI perspective could contribute to new applications and corresponding studies.

\subsection{In Relation to Robotics and Rehabilitation}

The fields of robotics and rehabilitation study a wide range of prostheses and design of neuromuscular control systems. A potential synergy between these fields and HCI can explore further use of such systems beyond exclusive scenarios. For example, the works presented in previous sections $[44,56,62,106]$, showcase new setups developed in light of computer-mediated interaction. The degree of usability of such systems can be evaluated and tuned through the interaction practices and methodologies already established in HCI.

\subsection{In Relation to Cognitive Science}

Cognitive and neuroscience research widely investigate the effect of embodiments on behaviors and cognitive processes. Understanding the depth of such functions will unveil further opportunities in computer-mediated communication, teleoperation, and wearable computers-topics actively discussed within HCI. Such understanding can not only affect the design rationales for systems, but could be actively connected with a body's sensory or nervous systems. For example, researchers $[107,108]$ have explored how rich haptic feedback for VR can be constructed by tricking the sense of orientation, or through a Wizard-of-Oz style reconfiguration of physical spaces.

In fact, cognitive science researchers are already pushing the boundaries, focusing not only on sociological factors [36-38] or neural phenomena [20-22], but also on parameters that are essential to designing an interaction system. For instance, the discussion by Aymerich-Franch and Ganesh [23] addresses the effect of (the body's) functionality in self-attribution. However, few works [17] have studied the relationship between embodiment and its interaction with the world or tasks. More critical interrogation towards the interaction aspect will lead to insights for designing a system that partially becomes a part of a user and its association with application contexts.

\subsection{Evaluation and Deployment}

Deployment and scaling of the technologies discussed in this article may be a challenge. A technology with very close proximity to the body, or one that is potentially invasive, has to be evaluated differently than conventional user interfaces. Reliability, robustness, running times and health effects will all be key parameters in the success of such technologies. For example, in the context of skin interfaces, the composition should always adhere to the body, should not fail in sensing, and should be free from any long-term health effects. This will demand another level of user studies than what is customary in $\mathrm{HCI}$ research, where elongated use of the technology has to be investigated. Similar knowledge has been generated by wearable research $[109,110]$ where studies have been mostly done through user surveys. HCI researchers have also explored the plausibility of implantable devices through tests on cadavers [111].

The field has attempted to find the means to explore these new ideas. However, there is still a long way to go before actual products will become available. It was only the last year (2016) when an automatic insulin injector was approved by the FDA [112]. Still, only 5\% of people among 29 million people in the US with diabetes [113] are eligible for the use of the technology. This is just one case that 
demonstrates that productisation of such technologies may take a long time, even if there is a large need. In the case of the new technologies discussed in the paper, deployment may take even longer, owing to the novelty of the concepts and applications.

\section{Conclusions}

In this paper, we discussed the concept of user interfaces that are closely integrated with the human body. As shown in numerous neuroscience and cognitive science studies, the use of tools affects how our brain functions. Building upon our human nature of incorporating tools as our extension, we envision computational interfaces that are internalized and augment our cognition and functional capabilities. We focused on three areas of augmentation, physical morphology, on-skin interfaces, and somatosensory extension, and presented examples of work by researchers and artists. In addition, recent developments in bioelectronics for body-implanted computers were discussed. The authors explored robotic and on-skin interfaces for transforming what the human body is and what it can do. While the research projects surveyed mostly focus on implementation, we believe future research in how such technologies can transform self-image and body-brain mapping will offer deeper insight into symbiotic user interfaces.

Acknowledgments: We thank all collaborators of projects demonstrated in this paper. DuoSkin and EarthTones are collaborations between MIT Media Lab and Microsoft Research; please refer to the papers for more information. The biosensor tattoos were developed by one of the authors Xin Liu in collaboration with Katia Vega. TreeSense is a collaboration between Xin Liu and Yedan Qian.

Author Contributions: All authors contributed to the overall conception of the scope of the paper. Sang-won Leigh managed the entire structure of the paper. Sang-won Leigh, Harpreet Sareen, Hsin-Liu Kao, and Xin Liu provided studies/materials on prior work. Sang-won Leigh, Hsin-Liu Kao, and Xin Liu also offered discussion on their on-going research progress.

Conflicts of Interest: The authors declare no conflict of interest.

\section{References}

1. Clynes, M.E.; Kline, N.S. Cyborgs and Space. In The Cyborg Handbook; Gray, C.H., Ed.; Routledge: London, UK, 1995; pp. 29-33.

2. Clark, A. Natural-Born Cyborgs: Minds, Technologies, and the Future of Human Intelligence. Oxford University Press: Oxford, UK, 2004.

3. Lakoff, G.; Johnson, M. Metaphors We Live By; University of Chicago Press: Chicago, IL, USA, 1980.

4. Lemley, B. Future Tech: Really Special Forces. Discover 2002, 2, 25-26.

5. Pratt, J.E.; Krupp, B.T.; Morse, C.J.; Collins, S.H. The RoboKnee: An Exoskeleton for Enhancing Strength and Endurance during Walking. In Proceedings of the IEEE International Conference on Robotics and Automation, ICRA '04, New Orleans, LA, USA, 26 April-1 May 2004; pp. 2430-2435.

6. Bundhoo, V.; Park, E.J. Design of an Artificial Muscle Actuated Finger towards Biomimetic Prosthetic Hands. In Proceedings of the 2005 IEEE International Conference on Robotics and Automation, ICAR '05, Barcelona, Spain, 18-22 April 2005; pp. 368-375.

7. Carrozza, M.C.; Massa, B.; Micera, S.; Lazzarini, R.; Zecca, M.; Dario, P. The Development of a Novel Prosthetic Hand-Ongoing Research and Preliminary Results. IEEE/ASME Trans. Mechatron. 2002, 7, 108-114. [CrossRef]

8. Popovic, M.; Popovic, D.; Tomovic, R. Control of Arm Movement: Reaching Synergies for Neuroprosthesis with Life-like Control. J. Autom. Control 2002, 12, 9-15. [CrossRef]

9. Nagel, S.K.; Carl, C.; Kringe, T.; Märtin, R.; König, P. Beyond Sensory Substitution—learning the Sixth Sense. J. Neural Eng. 2005, 2, R13-R26. [CrossRef] [PubMed]

10. Bach-y-Rita, P.; Collins, C.C.; Saunders, F.A.; White, B.; Scadden, L. Vision Substitution by Tactile Image Projection. Nature 1969, 221, 963-964. [CrossRef] [PubMed]

11. Shneiderman, B. Designing the User Interface; Pearson Education: Upper Saddle River, NJ, USA, 1998.

12. Clark, A. Embodied Prediction. In Open MIND; Metzinger, T.K., Windt, J.M., Eds.; MIND Group: Frankfurt, Germany, 2015. 
13. Wilson, M. Six Views of Embodied Cognition. Psychon. Bull. Rev. 2002, 9, 625-636. [CrossRef] [PubMed]

14. Lanier, J. Homuncular Flexibility. 2006. Available online: https://www.edge.org/response-detail/11182 (accessed on 7 March 2017).

15. Won, A.S.; Bailenson, J.N.; Lanier, J. Homuncular Flexibility: The Human Ability to Inhabit Nonhuman Avatars. In Emerging Trends in the Social and Behavioral Sciences; John Wiley \& Sons: Hoboken, NJ, USA, 2015; pp. 1-16.

16. Won, A.S.; Haans, A.; Ijsselsteijn, W.A.; Bailenson, J.N. A Framework for Interactivity and Presence in Novel Bodies. In Interacting with Presence: HCI and the Sense of Presence in Computer-mediated Environments; DE GRUYTER OPEN: Warsaw, Poland, 2014.

17. Won, A.S.; Bailenson, J.; Lee, J.; Lanier, J. Homuncular Flexibility in Virtual Reality. J. Comput. Commun. 2015, 20, 241-259. [CrossRef]

18. Wilson, F.R. The Hand: How Its Use Shapes the Brain, Language, and Human Culture; Pantheon Books: New York, NY, USA, 1998.

19. Marzke, M.W.; Toth, N.; Schick, K.; Reece, S.; Steinberg, B.; Hunt, K.; Linscheid, R.L.; An, K.N. EMG Study of Hand Muscle Recruitment during Hard Hammer Percussion Manufacture of Oldowan Tools. Am. J. Phys. Anthropol. 1998, 105, 315-332. [CrossRef]

20. Kilteni, K.; Normand, J.-M.; Sanchez-Vives, M.V.; Slater, M. Extending Body Space in Immersive Virtual Reality: A Very Long Arm Illusion. PLoS One 2012, 7, e40867. [CrossRef] [PubMed]

21. Iriki, A.; Tanaka, M.; Iwamura, Y. Coding of Modified Body Schema during Tool Use by Macaque Postcentral Neurones. Neuroreport 1996, 7, 2325-2330. [PubMed]

22. Berti, A.; Frassinetti, F. When Far Becomes near: Remapping of Space by Tool Use. J. Cogn. Neurosci. 2000, 12, 415-420. [CrossRef] [PubMed]

23. Aymerich-Franch, L.; Ganesh, G. The Role of Functionality in the Body Model for Self-Attribution. Neurosci. Res. 2016, 104, 31-37. [CrossRef] [PubMed]

24. Svanæs, D. Interaction Design for and with the Lived Body. ACM Trans. Comput. Interact. 2013, 20, 1-30. [CrossRef]

25. Gaver, W.W. Technology Affordances. In Proceedings of the SIGCHI Conference on Human Factors in Computing Systems, CHI '91, New Orleans, LA, USA, 27 April-2 May 1991; ACM Press: New York, NY, USA, 1991; pp. 79-84.

26. Gibson, J.J. The Ecological Approach to Visual Perception; Houghton Mifflin: Boston, MA, USA, 1979.

27. Heidegger, M. Being and Time; Harper \& Row: New York, NY, USA, 1962.

28. Merleau-Ponty, M. Phenomenology of Perception; Routledge \& Kegan Paul: London, UK, 1962.

29. Friston, K.J.; Daunizeau, J.; Kilner, J.; Kiebel, S.J. Action and Behavior: A Free-Energy Formulation. Biol. Cybern. 2010, 102, 227-260. [CrossRef] [PubMed]

30. Guterstam, A.; Petkova, V.I.; Ehrsson, H.H. The Illusion of Owning a Third Arm. PLoS One 2011, 6, e17208. [CrossRef] [PubMed]

31. Licklider, J.C.R. Man-Computer Symbiosis. IRE Trans. Hum. Factors Electron. 1960, HFE-1, 4-11. [CrossRef]

32. Ishii, H.; Ullmer, B. Tangible Bits: Towards Seamless Interfaces between People, Bits and Atoms. In Proceedings of the SIGCHI Conference on Human Factors in Computing Systems, CHI '97, San Jose, CA, USA, 30 April-3 May 2007; ACM Press: New York, NY, USA, 1997; pp. 234-241.

33. Gindrat, A.-D.; Chytiris, M.; Balerna, M.; Rouiller, E.M.; Ghosh, A. Use-Dependent Cortical Processing from Fingertips in Touchscreen Phone Users. Curr. Biol. 2015, 25, 109-116. [CrossRef] [PubMed]

34. Elbert, T.; Pantev, C.; Wienbruch, C.; Rockstroh, B.; Taub, E. Increased Cortical Representation of the Fingers of the Left Hand in String Players. Science 1995, 270, 305-307. [CrossRef] [PubMed]

35. Pantev, C.; Engelien, A.; Candia, V.; Elbert, T. Representational Cortex in Musicians. Plastic Alterations in Response to Musical Practice. Ann. N. Y. Acad. Sci. 2001, 930, 300-314. [CrossRef] [PubMed]

36. Lee, J.-E.R.; Nass, C.I.; Bailenson, J.N. Does the Mask Govern the Mind?: Effects of Arbitrary Gender Representation on Quantitative Task Performance in Avatar-Represented Virtual Groups. Cyberpsychol. Behav. Soc. Netw. 2014, 17, 248-254. [CrossRef] [PubMed]

37. Purvis, C.K.; Jones, M.; Bailey, J.O.; Bailenson, J.; Taylor, C.B. Developing a Novel Measure of Body Satisfaction Using Virtual Reality. PLoS One 2015, 10, e0140158. [CrossRef] [PubMed]

38. Rosenberg, R.S.; Baughman, S.L.; Bailenson, J.N. Virtual Superheroes: Using Superpowers in Virtual Reality to Encourage Prosocial Behavior. PLoS One 2013, 8, e55003. [CrossRef] [PubMed] 
39. Parietti, F.; Chan, K.; Asada, H.H. Bracing the Human Body with Supernumerary Robotic Limbs for Physical Assistance and Load Reduction. In Proceedings of the 2014 IEEE International Conference on Robotics and Automation, ICRA '14, Hong Kong, China, 31 May-7 June 2014; pp. 141-148.

40. Wu, F.Y.; Asada, H. Bio-Artificial Synergies for Grasp Posture Control of Supernumerary Robotic Fingers. In Robotics: Science and Systems; University of California: Berkeley, CA, USA, 2014.

41. Wu, F.Y.; Asada, H.H. "Hold-and-manipulate" with a Single Hand Being Assisted by Wearable Extra Fingers. In Proceedings of the 2015 IEEE International Conference on Robotics and Automation, ICRA '15, Seattle, WA, USA, 26-30 May 2015; pp. 6205-6212.

42. Shilkrot, R.; Huber, J.; Meng Ee, W.; Maes, P.; Nanayakkara, S.C. FingerReader: A Wearable Device to Explore Printed Text on the Go. In Proceedings of the 33rd Annual ACM Conference on Human Factors in Computing Systems, CHI '15, Seoul, Korea, 18-23 April 2015; ACM Press: New York, NY, USA, 2015; pp. 2363-2372.

43. Bau, O.; Popyrev, I.; Suzuki, Y. Ishin-Den-Shin. Available online: https://www.disneyresearch.com/project/ ishin-den-shin/ (accessed on 7 March 2017).

44. Tamaki, E.; Miyaki, T.; Rekimoto, J. PossessedHand: Techniques for Controlling Human Hands Using Electrical Muscles Stimuli. In Proceedings of the 2011 Annual Conference on Human Factors in Computing Systems, CHI '11, Vancouver, BC, Canada, 7-12 May 2011; ACM Press: New York, NY, USA, 2011; p. 543.

45. Lopes, P.; Jonell, P.; Baudisch, P. Affordance++: Allowing Objects to Communicate Dynamic Use. In'Proceedings of the 33rd Annual ACM Conference on Human Factors in Computing Systems, CHI '15, Seoul, Korea, 18-23 April 2015; ACM Press: New York, NY, USA, 2015; pp. 2515-2524.

46. Zoss, A.B.; Kazerooni, H.; Chu, A. Biomechanical Design of the Berkeley Lower Extremity Exoskeleton (BLEEX). IEEE/ASME Trans. Mechatron. 2006, 11, 128-138. [CrossRef]

47. Grabowski, A.M.; Herr, H.M. Leg Exoskeleton Reduces the Metabolic Cost of Human Hopping. J. Appl. Physiol. 2009, 107, 670-678. [CrossRef] [PubMed]

48. Herr, H.; Gamow, R.I. Shoe and Foot Prosthesis with Bending Beam Spring Structures. U.S. Patent 5,701,686, 29 February 2000.

49. PowerSkip. Available online: http:/ / www.powerskip.de (accessed on 7 March 2017).

50. Dick, J.; Edwards, E. Human Bipedal Locomotion Device. U.S. Patent 5,016,869, 21 May 1991.

51. Herr, H. Exoskeletons and Orthoses: Classification, Design Challenges and Future Directions. J. Neuroeng. Rehabil. 2009, 6, 21. [CrossRef] [PubMed]

52. Linn, D.M.; Ihrke, C.A.; Diftler, M.A. Human Grasp Assist Device and Method of Use. U.S. Patent 8,255,079 B2, 28 August 2012.

53. Hawkes, E.W.; Jiang, H.; Cutkosky, M.R. Three-Dimensional Dynamic Surface Grasping with Dry Adhesion. Int. J. Rob. Res. 2016, 35, 943-958. [CrossRef]

54. Bonilla, B.L.; Asada, H.H. A Robot on the Shoulder: Coordinated Human-Wearable Robot Control Using Coloured Petri Nets and Partial Least Squares Predictions. In Proceedings of the 2014 IEEE International Conference on Robotics and Automation, ICRA '14, Hong Kong, China, 31 May-7 June 2014; pp. 119-125.

55. Parietti, F.; Chan, K.C.; Hunter, B.; Asada, H.H. Design and Control of Supernumerary Robotic Limbs for Balance Augmentation. In Proceedings of the 2015 IEEE International Conference on Robotics and Automation, ICRA '15, Seattle, WA, USA, 26-30 May 2015; pp. 5010-5017.

56. Gopinath, D.; Weinberg, G. A Generative Physical Model Approach for Enhancing the Stroke Palette for Robotic Drummers. Rob. Auton. Syst. 2016, 86, 207-215. [CrossRef]

57. Stelarc. Exoskeleton. Available online: http: / / stelarc.org/ ?catID=20227 (accessed on 7 March 2017).

58. Demers, L.P.; Vorn, B. Inferno. Available online: https://vimeo.com/130670526 (accessed on 7 March 2017).

59. Hameed, J.; Harrison, I.; Gasson, M.N.; Warwick, K. A Novel Human-Machine Interface Using Subdermal Magnetic Implants. In Proceedings of the 2010 IEEE 9th International Conference on Cyberntic Intelligent Systems, Reading, UK, 1-2 September 2010; pp. 1-5.

60. Haans, A.; IJsselsteijn, W.A. Embodiment and Telepresence: Toward a Comprehensive Theoretical Framework. Interact. Comput. 2012, 24, 211-218. [CrossRef]

61. Project Cyborg. Available online: http://www.kevinwarwick.com/project-cyborg-1-0/ (accessed on 7 March 2017). 
62. Leigh, S.; Maes, P. Body Integrated Programmable Joints Interface. In Proceedings of the $2016 \mathrm{CHI}$ Conference Extended Abstracts on Human Factors in Computing Systems, CHI EA '16, San Jose, CA, USA, 7-12 May 2016; ACM Press: New York, NY, USA; pp. 3719-3722.

63. Farahi, B. Caress of the Gaze. Available online: http://behnazfarahi.com/caress-of-the-gaze (accessed on 7 March 2017).

64. Kao, H.-L.; Holz, C.; Roseway, A.; Calvo, A.; Schmandt, C. DuoSkin: Rapidly Prototyping on-Skin User Interfaces Using Skin-Friendly Materials. In Proceedings of the 2016 ACM International Symposium on Wearable Computers, ISWC '16, Heidelberg, Germany, 12-16 September 2016; ACM Press: New York, NY, USA, 2016; pp. 16-23.

65. Liu, X.; Vega, K.; Maes, P.; Paradiso, J.A. Wearability Factors for Skin Interfaces. In Proceedings of the 7th Augmented Human International Conference, AH '16, Geneva, Switzerland, 25-27 February 2016; ACM Press: New York, NY, USA, 2016; pp. 1-8.

66. Lo, J.; Lee, D.J.L.; Wong, N.; Bui, D.; Paulos, E. Skintillates. In Proceedings of the 2016 ACM Conference on Designing Interactive Systems, DIS '16, Brisbane, Australia, 4-8 June 2016; ACM Press: New York, NY, USA, 2016; pp. 853-864.

67. El Kaliouby, R.; Teeters, A.; Picard, R.W. An Exploratory Social-Emotional Prosthetic for Autism Spectrum Disorders. In Proceedings of the International Workshop on Wearable and Implantable Body Sensor Networks (BSN'06), 3-5 April 2006; pp. 3-4.

68. Bardzell, J.; Bardzell, S.; Stolterman, E. Reading Critical Designs. In Proceedings of the 32nd Annual ACM Conference on Human Factors in Computing Systems, CHI '14, Toronto, ON, Canada, 26 April-1 May 2014; ACM Press: New York, NY, USA, 2014; pp. 1951-1960.

69. Yoon, M. Defensible Dress. Available online: http://www.mystudio.us/projects/defensible-dress (accessed on 7 March 2017).

70. Anouk Wipprecht Homepage. Available online: http://www.anoukwipprecht.nl/\#intro (accessed on 7 March 2017).

71. Ying Gao Homepage. Available online: http://yinggao.ca/interactifs/incertitudes/ (accessed on 7 March 2017).

72. Liu, A.; Lengeling, T. Reflesh. Available online: http://ani-liu.com/reflesh (accessed on 7 March 2017).

73. Bechara, A.; Damasio, H.; Tranel, D.; Damasio, A.R. The Iowa Gambling Task and the somatic marker hypothesis: Some questions and answers. Trends Cogn. Sci. 2005, 9, 159-162. [CrossRef] [PubMed]

74. Harrison, C.; Tan, D.; Morris, D. Skinput: appropriating the body as an input surface. In Proceedings of The 28th International Conference on Human Factors in Computing Systems, CHI '10, Atlanta, GA, USA, 10-15 April 2010; ACM Press: New York, NY, USA, 2010; p. 453.

75. Kao, H.-L.; Dementyev, A.; Paradiso, J.A.; Schmandt, C. NailO: Fingernails as an Input Surface. In Proceedings of the 33rd Annual ACM Conference on Human Factors in Computing Systems, CHI '15, Seoul, Korea, 18-23 April 2015; ACM Press: New York, NY, USA, 2015; pp. 3015-3018.

76. Weigel, M.; Lu, T.; Bailly, G.; Oulasvirta, A.; Majidi, C.; Steimle, J. iSkin: Flexible, Stretchable and Visually CustomizableOn-Body Touch Sensors for Mobile Computing. In Proceedings of the 33rd Annual ACM Conference on Human Factors in Computing Systems, CHI '15, Seoul, Korea, 18-23 April 2015; ACM Press: New York, NY, USA, 2015; pp. 2991-3000.

77. Chan, L.; Liang, R.-H.; Tsai, M.-C.; Cheng, K.-Y.; Su, C.-H.; Chen, M.Y.; Cheng, W.-H.; Chen, B.-Y. FingerPad: Private and Subtle Interaction Using Fingertips. In Proceedings of the 26th Annual ACM Symposium on User Interface Software and Technology, UIST '13, St Andrews, UK, 8-11 October 2013; ACM Press: New York, NY, USA, 2013; pp. 255-260.

78. Su, C.-H.; Chan, L.; Weng, C.-T.; Liang, R.-H.; Cheng, K.-Y.; Chen, B.-Y. NailDisplay: Bringing an Always Available Visual Display to Fingertips. In Proceedings of the SIGCHI Conference on Human Factors in Computing Systems, CHI '13, Paris, France, 27 April-2 May 2013; ACM Press: New York, NY, USA, 2013; p. 1461.

79. Vivalnk Tattoo. Available online: http://www.vivalnk.com/tattoo (accessed on 7 March 2017).

80. Kim, D.-H.; Lu, N.; Ma, R.; Kim, Y.-S.; Kim, R.-H.; Wang, S.; Wu, J.; Won, S.M.; Tao, H.; Islam, A.; et al. Epidermal Electronics. Science 2011, 333, 838-843. [CrossRef] [PubMed]

81. In Ten Years, You Won't Even Know You're Wearing Them. Available online: http://pbs.org/wgbh/nova/ next/tech/wearable-health-sensors/ (accessed on 7 March 2017). 
82. Would You Continue to Wear a Wearable Device If It Saves Your Life? Available online: http:/ / www.wearabletechworld.com/topics/wearable-tech/articles/385003-would-continue-wearwearable-device-if-it-saves.html (accessed on 7 March 2017).

83. Kao, H.L.; Nguyen, B.; Roseway, A.; Dickey, N. EarthTones: Chemical Sensing Powders to Detect and Display Environmental Hazards through Color Variation. In Proceedings of the alt.CHI, Denver, CO, USA, 6-11 May 2017; ACM Press: New York, NY, USA, 2017.

84. Critchley, H.D.; Wiens, S.; Rotshtein, P.; Ohman, A.; Dolan, R.J. Neural systems supporting interoceptive awareness. Nat. Neurosci. 2004, 7, 189-195. [CrossRef] [PubMed]

85. Wells, A.; Papageorgiou, C. Social phobic interoception: Effects of bodily information on anxiety, beliefs and self-processing. Behav. Res. Ther. 2001, 39,1-11. [CrossRef]

86. Wald, J.; Taylor, S. Responses to interoceptive exposure in people with posttraumatic stress disorder (PTSD): A preliminary analysis of induced anxiety reactions and trauma memories and their relationship to anxiety sensitivity and PTSD symptom severity. Cogn. Behav. Ther. 2008, 37, 90-100. [CrossRef] [PubMed]

87. Lee, K.; Noda, Y.; Nakano, Y.; Ogawa, S.; Kinoshita, Y.; Funayama, T.; Furukawa, T.A. Interoceptive Hypersensitivity and Interoceptive Exposure in Patients with Panic Disorder: Specificity and Effectiveness. BMC Psychiatry 2006, 6, 32. [CrossRef] [PubMed]

88. Craske, M.G.; Wolitzky-Taylor, K.B.; Labus, J.; Wu, S.; Frese, M.; Mayer, E.A.; Naliboff, B. A Cognitive-Behavioral Treatment for Irritable Bowel Syndrome Using Interoceptive Exposure to Visceral Sensations. Bhav. Res. Ther. 2011, 49, 413-421. [CrossRef] [PubMed]

89. Suzuki, K.; Garfinkel, S.N.; Critchley, H.D.; Seth, A.K. Multisensory Integration across Exteroceptive and Interoceptive Domains Modulates Self-Experience in the Rubber-Hand Illusion. Neuropsychologia 2013, 51, 2909-2917. [CrossRef] [PubMed]

90. Banissy, M.J.; Cohen Kadosh, R.; Maus, G.W.; Walsh, V.; Ward, J. Prevalence, characteristics and a neurocognitive model of mirror-touch synaesthesia. Exp. Brain Res. 2009, 198, 261-272. [CrossRef] [PubMed]

91. Tajadura-Jiménez, A.; Väljamäe, A.; Toshima, I.; Kimura, T.; Tsakiris, M.; Kitagawa, N. Action sounds recalibrate perceived tactile distance. Curr. Biol. 2012, 22, R516-R517. [CrossRef] [PubMed]

92. Tajadura-Jiménez, A.; Grehl, S.; Tsakiris, M. The other in me: interpersonal multisensory stimulation changes the mental representation of the self. PLoS ONE 2012, 7, e40682. [CrossRef] [PubMed]

93. BeAnotherLab. Available online: http:/ / beanotherlab.org/ (accessed on 7 March 2017).

94. Liu, X.; Qian, Y. TreeSense. Available online: https://www.media.mit.edu/projects/treesense/overview/ (accessed on 7 March 2017).

95. Adamatzky, A. Physarum Machines; World Scientific: Singapore, 2010.

96. OpenWorm. Available online: http:/ /www.openworm.org/ (accessed on 7 March 2017).

97. Fukayama, O.; Nakanishi, R.; Otsuka, H.; Suzuki, T.; Mabuchi, K. RatCar: A Whole-Body Neuromuscular Locomotion Prosthetic Device with Exoskeletal Robotic Limbs for a Rat. In Proceedings of The 6th International Conference on Soft Computing and Intelligent Systems, and The 13th International Symposium on Advanced Intelligence Systems, Kobe, Japan, 20-24 November 2012; pp. 1158-1161.

98. Bakkum, D.J.; Booth, M.C.; Brumfield, J.R.; Chao, Z.; Madhavan, R.; Passaro, P.A.; Rambani, K.; Shkolnik, A.C.; Towal, R.B. Hybrots: Hybrids of living neurons and robots for studying neural computation. In Proceedings of the Brain Inspired Cognitive Systems, Stirling, UK, 29 August-1 September 2004.

99. Velliste, M.; Perel, S.; Spalding, M.C.; Whitford, A.S.; Schwartz, A.B. Cortical Control of a Prosthetic Arm for Self-Feeding. Nature 2008, 453, 1098-1101. [CrossRef] [PubMed]

100. Schnell, E.; Klinkhammer, K.; Balzer, S.; Brook, G.; Klee, D.; Dalton, P.; Mey, J. Guidance of Glial Cell Migration and Axonal Growth on Electrospun Nanofibers of Poly-Epsilon-Caprolactone and a Collagen/poly-Epsilon-Caprolactone Blend. Biomaterials 2007, 28, 3012-3025. [CrossRef] [PubMed]

101. Ortiz-Catalan, M.; Hakansson, B.; Branemark, R. An Osseointegrated Human-Machine Gateway for Long-Term Sensory Feedback and Motor Control of Artificial Limbs. Sci. Transl. Med. 2014, 6, 257. [CrossRef] [PubMed]

102. Navarro, X.; Krueger, T.B.; Lago, N.; Micera, S.; Stieglitz, T.; Dario, P. A Critical Review of Interfaces with the Peripheral Nervous System for the Control of Neuroprostheses and Hybrid Bionic Systems. J. Peripher. Nerv. Syst. 2005, 10, 229-258. [CrossRef] [PubMed] 
103. Kameswaran, N.; Cullen, D.K.; Pfister, B.J.; Ranalli, N.J.; Huang, J.H.; Zager, E.L.; Smith, D.H. A Novel Neuroprosthetic Interface with the Peripheral Nervous System Using Artificially Engineered Axonal Tracts. Neurol. Res. 2008, 30, 1063-1067. [CrossRef] [PubMed]

104. Smith, D.H.; Wolf, J.A.; Meaney, D.F. A New Strategy to Produce Sustained Growth of Central Nervous System Axons: Continuous Mechanical Tension. Tissue Eng. 2001, 7, 131-139. [CrossRef] [PubMed]

105. Kuiken, T.A. Targeted Muscle Reinnervation for Real-Time Myoelectric Control of Multifunction Artificial Arms. JAMA 2009, 301, 619. [CrossRef] [PubMed]

106. Lopes, P.; Yüksel, D.; Guimbretière, F.; Baudisch, P. Muscle-Plotter: An Interactive System Based on Electrical Muscle Stimulation That Produces Spatial Output. In Proceedings of the 29th Annual Symposium on User Interface Software and Technology, UIST '16, Tokyo, Japan, 16-19 October 2016; ACM Press: New York, NY, USA, 2016; pp. 207-217.

107. Cheng, L.-P.; Roumen, T.; Rantzsch, H.; Köhler, S.; Schmidt, P.; Kovacs, R.; Jasper, J.; Kemper, J.; Baudisch, P. TurkDeck: Physical Virtual Reality Based on People. In Proceedings of the 28th Annual ACM Symposium on User Interface Software \& Technology, UIST '15, Charlotte, NC, USA, 11-15 November 2015; ACM Press: New York, NY, USA, 2015; pp. 417-426.

108. Azmandian, M.; Hancock, M.; Benko, H.; Ofek, E.; Wilson, A.D. Haptic Retargeting: Dynamic Repurposing of Passive Haptics for Enhanced Virtual Reality Experiences. In Proceedings of the 2016 CHI Conference on Human Factors in Computing Systems, CHI '16, San Jose, CA, USA, 7-12 May 2016; ACM Press: New York, NY, USA, 2016; pp. 1968-1979.

109. Clawson, J.; Pater, J.A.; Miller, A.D.; Mynatt, E.D.; Mamykina, L. No Longer Wearing: Investigating the Abandonment of Personal Health-Tracking Technologies on Craigslist. In Proceedings of the 2015 ACM International Joint Conference on Pervasive and Ubiquitous Computing, UbiComp '15, Osaka, Japan, 7-11 September 2015; ACM Press: New York, NY, USA, 2015; pp. 647-658.

110. Lazar, A.; Koehler, C.; Tanenbaum, J.; Nguyen, D.H. Why We Use and Abandon Smart Devices. In Proceedings of the 2015 ACM International Joint Conference on Pervasive and Ubiquitous Computing, UbiComp '15, Osaka, Japan, 7-11 September 2015; ACM Press: New York, NY, USA, 2015; pp. 635-646.

111. Holz, C.; Grossman, T.; Fitzmaurice, G.; Agur, A. Implanted User Interfaces. In Proceedings of the 2012 ACM Annual Conference on Human Factors in Computing Systems, CHI '12, Austin, TX, USA, 5-10 May 2012; ACM Press: New York, NY, USA, 2012; p. 503.

112. FDA approves first automated insulin delivery device for type 1 diabetes. Available online: http://www. fda.gov/NewsEvents/Newsroom/PressAnnouncements/ucm522974.htm (accessed on 7 March 2017).

113. What is Type 1 Diabetes (T1D)? Available online: http://www.jdrf.org/about/about-type-1-diabetes-t1d/ (accessed on 7 March 2017). 